\title{
Essential medicines for children: an endocrine perspective
}

\author{
Sanjay Kalra ${ }^{1 * *}$, Yashdeep Gupta ${ }^{2}$
}

\begin{abstract}
*Correspondence to: Sanjay Kalra, Email: brideknl@gmail.com Copyright: ( 2014 by Kerman University of Medical Sciences Citation: Kalra S, Gupta Y. Essential medicines for children: an endocrine perspective. Int J Health Policy Manag 2014; 3: 357. doi: 10.15171/ijhpm.2014.112 Received: 18 October 2014, Accepted: 28 October 2014, ePublished: 29 October 2014
\end{abstract}

\section{Dear Editor,}

The emergence of endocrine disease has created significant challenges for healthcare policy-makers and payers across the world. Policy-makers have to ensure availability of drugs used for various endocrinopathies. One way in which this is facilitated is through the World Health Organization (WHO) List of Essential Medicines (LEM). The LEM aims to cover the basic pharmaceutical needs of the majority of people seeking healthcare (1).

The unique nature of paediatric health is well-known. The WHO's Model LEM for Children, issued in April 2013, covers the needs of children up to 12 years of age (2). In its core list, it includes drugs for priority conditions, which "are selected on the basis of current and estimate future public health relevance and potential for safe and cost-effective treatment". The complementary list includes important medicinal drugs which do not necessarily fit the above definition, but are required in routine clinical practice.

The list enumerates various "hormones, other endocrine drugs and contraceptives". Levothyroxine is included as a core medicine in strengths of 25,50 and $100 \mathrm{mcg}$. The complementary list includes Lugol's iodine, potassium iodide, and propylthiouracil (2). Adrenal hormones included are oral fludrocortisone and hydrocortisone (2). Diabetes is covered by soluble insulin, and intermediate-acting insulin (compound insulin zinc suspension or isophane insulin). Metformin is listed as a complementary medicine, while glucagon $1 \mathrm{mg} / \mathrm{ml}$ is included in the core list (2). A separate section on 'vitamins and minerals' lists liquid (400 IU/ml) and solid dosage forms (400 IU, 1,000 IU) of cholecalciferol, while mentioning ergocalciferol as an alternative (2).

From an endocrine perspective, the inclusion of these drugs is laudable. Paediatric thyroid disorders are increasing in prevalence, and adrenal disorders, though not as common, are life threatening if not treated. The diabetes pandemic has not spared children and insulin, a life-saving drug, is mandatory in any paediatric LEM. The mention of glucagon, an emergency drug for management of hypoglycemia, is praise worthy. A few omissions, though, need to be highlighted. No pituitary drug (growth hormone) is mentioned in the LEM. Similarly methimazole or carbimazole, which are safe anti thyroid drugs, are not included. The diabetes pharmacotherapeutic landscape is very vast. The current LEM includes human insulins, and allows basal-bolus and split-mix regimes be used. These are difficult to follow in primary care practice, and premixed insulin may be needed in some settings (3). 'Compound insulin zinc' is no longer manufactured, and should be deleted (4). The advantages of insulin analogues (lower risk of hypoglycemia) are well-documented (5). Therefore, insulin analogues should be added as complementary drugs. Metformin, an economical drug, frequently used in paediatrics, (6) deserves a shift to the core list. A relook at the omission of calcium is necessary, too. The WHO Model LEM for Children provides a framework for ensuring availability of essential drugs across the world. With the increase in prevalence of paediatric endocrine morbidity, renewed emphasis on the inclusion of appropriate endocrine drugs in the WHO LEM for Children is necessary.

\section{Ethical issues \\ Not applicable.}

Competing interests

Authors declare that they have no competing interests.

Authors' contributions

Both authors have contributed equally to the manuscript.

\section{References}

1. World Health Organization (WHO) Model List of Essential Medicines [internet]. [cited 2014 October 08]. Available from: http://www.who.int/medicines/publications/essentialmedicines/ en/index.html

2. World Health Organization (WHO) Model List of Essential Medicines for Children [internet]. [cited 2014 October 08]. Available from: http://www.who.int/medicines/publications/ essentialmedicines/4th_EMLC_FINAL_web_8Jul13.pdf

3. de Beaufort CE, Lange K, Swift PG, Aman J, Cameron F, Castano L, et al. Metabolic outcomes in young children with type 1 diabetes differ between treatment centers: the Hvidoere Study in Young Children 2009. Pediatr Diabetes 2013; 14: 422-8. doi: 10.1111/j.1399-5448.2012.00922.x

4. Kalra S, Bajaj S, Mithal A. Endocrinology and the essential list. Indian J Endocr Metab 2012; 16: 133-5.

5. Pedersen-Bjergaard $U$, Kristensen $\mathrm{PL}$, Beck-Nielsen $\mathrm{H}$, Nørgaard K, Perrild H, Christiansen JS. Effect of insulin analogues on risk of severe hypoglycaemia in patients with type 1 diabetes prone to recurrent severe hypoglycaemia (HypoAna trial): a prospective, randomised, open-label, blinded-endpoint crossover trial. Lancet Diabetes Endocrinol 2014; 2: 553-61. doi: 10.1016/S2213-8587(14)70073-7

6. Konrad K, Datz N, Engelsberger I, Grulich-Henn J, Hoertenhuber $\mathrm{T}$, Knauth $\mathrm{B}$, et al. Current use of metformin in addition to insulin in pediatric patients with type 1 diabetes mellitus: an analysis based on a large diabetes registry in Germany and Austria. Pediatr Diabetes 2014. doi: 10.1111/pedi.12203 